\title{
ADDITIVE COMPARISONS OF STOP RULE AND SUPREMUM EXPECTATIONS OF UNIFORMLY BOUNDED INDEPENDENT RANDOM VARIABLES
}

\author{
T. P. HILL ${ }^{1}$ AND ROBERT P. KERTZ
}

\begin{abstract}
Let $X_{1}, X_{2}, \ldots$ be independent random variables taking values in $[a, b]$, and let $T$ denote the stop rules for $X_{1}, X_{2}, \ldots$ Then $E\left(\sup _{n>1} X_{n}\right)-$ $\sup \left\{E X_{t}: t \in T\right\}<(1 / 4)(b-a)$, and this bound is best possible. Probabilistically, this says that if a prophet (player with complete foresight) makes a side payment of $(b-a) / 8$ to a gambler (player using nonanticipating stop rules), the game becomes at least fair for the gambler.
\end{abstract}

1. Introduction. Suppose that $X_{1}, X_{2}, \ldots$ are independent nonnegative random variables on a probability space $(\Omega, \mathfrak{A}, P)$, and let $T_{n}$ denote the stop rules for $X_{1}, \ldots, X_{n}$ and $T$ denote the stop rules for $X_{1}, X_{2}, \ldots$ The inequality $E\left(\max \left\{X_{1}, \ldots, X_{n}\right\}\right) \leqslant k \sup \left\{E X_{t}: t \in T_{n}\right\}$ has been studied in the theory of semiamarts where Krengel and Sucheston [3] discovered that $k$ can always be taken $<4$. Garling's proof [4] showed that $k=2$, and that 2 is the best possible universal bound, and Hill and Kertz [2] found that in all nontrivial situations, weak inequality actually holds.

Such comparisons of expectations of the maximum with optimal stop rule expectations have been interpreted in probabilistic terms as comparisons between the optimal expected return of a prophet (a player with complete foresight), and a gambler (player using only nonanticipating stop rules). In this language, the $k=2$ result says that the odds 2:1 make the game at least favorable for the gambler (versus a prophet playing the same game).

The purpose of this paper is to study the difference $E\left(\sup _{n>1} X_{n}\right)-\sup \left\{E X_{t}\right.$ : $t \in T\}$ in the case the $\left\{X_{i}\right\}$ are uniformly bounded. The main result is

THEOREM A. If $X_{1}, X_{2}, \ldots$ are independent random variables taking values in $[a, b]$, then $E\left(\sup _{n>1} X_{n}\right)-\sup \left\{E X_{t}: t \in T\right\}<(1 / 4)(b-a)$ (equivalently $\left.\inf \left\{E X_{t}: t \in T\right\}-E\left(\inf _{n>1} X_{n}\right) \leqslant(1 / 4)(b-a)\right)$, and the bound is best possible.

This result may be interpreted probabilistically as saying that in a uniformly bounded situation, a side payment, from a prophet to a gambler, of $(b-a) / 8$ makes the game at least fair for the gambler.

Received by the editors September 11, 1980 and, in revised form, January 24, 1981; presented to the Society at the 87th Annual Meeting, January 10, 1981.

1980 Mathematics Subject Classification. Primary 60G40; Secondary 62L15, 90C99.

${ }^{1}$ Partially supported by AFOSR Grant F49620-C-0123. 
2. Preliminaries. For random variables $X$ and $Y, X \vee Y$ denotes the maximum of $X$ and $Y, X^{+}$is $X \vee 0$, and $E X$ is the expectation of $X$. The value $V\left(X_{1}, \ldots, X_{n}\right)$ of an ordered collection of independent random variables $X_{1}, \ldots, X_{n}$ is defined to be $V\left(X_{1}, \ldots, X_{n}\right)=\sup \left\{E X_{t}: t\right.$ is a stop rule for $\left.X_{1}, \ldots, X_{n}\right\}$.

For ease of reference, we include the following lemma, a consequence of backward induction.

LEMMA $2.1\left(\left[1\right.\right.$, p. 50]). Let $X_{1}, \ldots, X_{n}$ be independent random variables. Then

(a) $V\left(X_{j}, \ldots, X_{n}\right)=E\left(X_{j} \vee V\left(X_{j+1}, \ldots, X_{n}\right)\right)$ for $j=1, \ldots, n-1$; and

(b) if $t^{*}$ is the stop rule defined by $t^{*}=j \Leftrightarrow\left\{t^{*}>j-1\right.$ and $X_{j}>$ $\left.V\left(X_{j+1}, \ldots, X_{n}\right)\right\}$, then $E X_{t^{*}}=V\left(X_{1}, \ldots, X_{n}\right)$.

Definition 2.2. For an integrable random variable $Y$ and constants $-\infty<a<b$ $<\infty$, let $Y_{a}^{b}$ denote a random variable with $Y_{a}^{b}=Y$ if $Y \notin[a, b],=a$ with probability $(b-a)^{-1} \int_{Y \in[a, b]}(b-Y)$, and $=b$ otherwise (i.e., $=b$ with probability $\left.(b-a)^{-1} \int_{Y \in[a, b]}(Y-a)\right)$.

The random variable $Y_{a}^{b}$ is extremal with respect to $Y, a$, and $b$ in the following sense, which is fundamental to the results in this paper.

LEMMA 2.2. Let $Y$ be any integrable random variable and $-\infty<a<b<\infty$. Then $E Y=E Y_{a}^{b}$, and if $X$ is any integrable random variable independent of both $Y$ and $Y_{a}^{b}$, then $E(X \vee Y)<E\left(X \vee Y_{a}^{b}\right)$.

Proof. That $E Y=E Y_{a}^{b}$ is immediate. For the second part of the conclusion, fix any $X$ independent of both $Y$ and $Y_{a}^{b}$, and verify that the function $\psi(y)=$ $E(X \vee y)$ is convex. From the independence of $X$ and $Y$, and convexity of $\psi$, it follows that

$$
\begin{aligned}
\int_{Y \in[a, b]}(X \vee Y)<(b-a)^{-1}\left\{[E(X \vee a)] \int_{Y \in[a, b]}(b-Y)\right. \\
\left.+[E(X \vee b)] \int_{Y \in[a, b]}(Y-a)\right\}
\end{aligned}
$$

Thus

$$
\begin{aligned}
E(X \vee Y) & =\int_{Y \notin[a, b]}(X \vee Y)+\int_{Y \in[a, b]}(X \vee Y) \\
& <\int_{Y \notin[a, b]} X \vee Y+(b-a)^{-1}\left\{[E(X \vee a)] \int_{Y \in[a, b]}(b-Y)\right. \\
& \left.+[E(X \vee b)] \int_{Y \in[a, b]}(Y-a)\right\} \\
& =E\left(X \vee Y_{a}^{b}\right),
\end{aligned}
$$

where the inequality follows from (1).

An alternative characterization for the extremal random variable $Y_{a}^{b}$ is the following: $Y_{a}^{b}$ is the random variable with maximum variance which coincides with $Y$ off $[a, b]$, and which has expectation $E Y$. In this respect, the conclusion of Lemma 2.2 becomes rather intuitive. 
3. Proof of Theorem A. Without loss of generality (add, or multiply by, suitable constants) it will be assumed throughout the remainder of this paper that all random variables take values in $[0,1]$.

Definition 3.1. For random variables $X_{1}, \ldots, X_{n}$, define $D\left(X_{1}, \ldots, X_{n}\right)$, the additive advantage of the prophet over the gambler, by $D\left(X_{1}, \ldots, X_{n}\right)=$ $E\left(X_{1} \vee \cdots \vee X_{n}\right)-V\left(X_{1}, \ldots, X_{n}\right)$.

As in [2], the main step in the proof will be to show constructively that for any sequence of $n>2$ random variables there is a sequence of $n-1$ random variables offering at least as large an additive advantage to the prophet.

LEMMA 3.1. Given $n>2$ and independent r.v.'s $X_{1}, \ldots, X_{n}$, there exists a zeroone valued random variable $W$ independent of $X_{2}, \ldots, X_{n-2}$, and satisfying $D\left(X_{1}, \ldots, X_{n}\right) \leqslant D\left(\mu, X_{2}, \ldots, X_{n-2}, W\right)$, where $\mu=V\left(X_{2}, \ldots, X_{n}\right)$.

Proof. By Lemma 2.1, $V\left(X_{1}, \ldots, X_{n}\right)=V\left(\mu, X_{2}, \ldots, X_{n}\right)+E\left(X_{1}-\mu\right)^{+}$. Since $E\left(X_{1} \vee \cdots \vee X_{n}\right) \leqslant E\left(\mu \vee X_{2} \vee \cdots \vee X_{n}\right)+E\left(X_{1}-\mu\right)^{+}$, it follows that

$$
D\left(X_{1}, \ldots, X_{n}\right) \leqslant D\left(\mu, X_{2}, \ldots, X_{n}\right) .
$$

Let $Z=\left(X_{n}\right)_{0}^{1}$ and $Y=\left(X_{n-1}\right)_{E X_{n}}^{1}$ be independent of each other and of $X_{1}, \ldots, X_{n-2}$. By Lemmas 2.1 and 2.2, $V(Y, Z)=V\left(X_{n-1}, X_{n}\right)$, and therefore $V\left(\mu, X_{2}, \ldots, X_{n}\right)=V\left(\mu, X_{2}, \ldots, X_{n-2}, Y, Z\right)$. By Lemma 2.2,

$$
E\left(\mu \vee X_{2} \vee \cdots \vee X_{n}\right) \leqslant E\left(\mu \vee X_{2} \vee \ldots \vee X_{n-2} \vee Y \vee Z\right)
$$

Thus

$$
D\left(\mu, X_{2}, \ldots, X_{n}\right) \leqslant D\left(\mu, X_{2}, \ldots, X_{n-2}, Y, Z\right) .
$$

Let $W$ be any random variable independent of $X_{2}, \ldots, X_{n-2}$, and satisfying $P(W=1)=V(Y, Z)=1-P(W=0)$. Since $E W=V(Y, Z)$, and since $E Z=$ $E X_{n} \leqslant \mu$, it follows from Lemma 2.1 and the definitions of $Y, Z$, and $W$ that

$$
D\left(\mu, X_{2}, \ldots, X_{n-2}, Y, Z\right)=D\left(\mu, X_{2}, \ldots, X_{n-2}, W\right) \text {. }
$$

Combining (2), (3), and (4) completes the proof.

Proof of Theorem A. It is clear that it suffices to prove the result for a finite number of random variables (e.g., see [4, p. 237]), and, by Lemma 3.1, the proof is further reduced to showing that $D\left(X_{1}, X_{2}\right) \leqslant 1 / 4$, and that the bound is sharp. Letting $E X_{2}=\mu$, it follows as in (2) and (3) that $D\left(X_{1}, X_{2}\right)<D(\mu, Z)$, where $P(Z=1)=\mu=1-P(Z=0)$. But $D(\mu, Z)=E(\mu \vee Z)-V(\mu, Z)=$ $(\mu+(1-\mu) \mu)-\mu=\mu-\mu^{2} \leqslant 1 / 4$ for $\mu \in[0,1]$, and the bound $D=1 / 4$ is attained for $\mu=1 / 2$.

4. Remarks. The parenthetical conclusion in Theorem $A$ that $\inf \left\{E X_{t}: t \in T\right\}-$ $E\left(\inf _{n>1} X_{n}\right) \leqslant(1 / 4)(b-a)$ is immediate by symmetry. In contrast, no corresponding universal constant exists for ratio comparisons of $E\left(\min \left\{X_{1}, \ldots, X_{n}\right\}\right)$ and $\inf \left\{E X_{t}: t \in T_{n}\right\}$, even if the random variables are indentically distributed as well as uniformly bounded, as the following example shows.

EXAMPLE 4.1. Fix $n>1$ and $0<p<1 / 2$, and let $X_{1}, X_{2}, \ldots, X_{n}$ be i.i.d. each with common distribution given by $X_{i}=0$ with probability $1-p-p^{2},=$ $p^{2} /(1-p)$ with probability $p$, and $=1$ otherwise. Then 


$$
E\left(\min \left\{X_{1}, \ldots, X_{n}\right\}\right)=p^{2}\left[\left(p+p^{2}\right)^{n}-p^{2 n}+(1-p) p^{2 n-2}\right] /(1-p),
$$

and $\inf \left\{E X_{t}: t \in T_{n}\right\}=p^{2}\left(p+p^{2}\right)^{\mathrm{n}-1} /(1-\mathrm{p})$. For any $M>0$, and $p$ sufficiently small, the random variables $X_{1}, \ldots, X_{n}$ satisfy $\inf \left\{E X_{t}: t \in T_{n}\right\}>$ $\operatorname{ME}\left(\min \left\{X_{1}, \ldots, X_{n}\right\}\right)$.

If the independence assumption in Theorem $A$ is dropped, the conclusion may fail, even if the sequence $X_{1}, X_{2}, \ldots$ is both a martingale and Markovian.

EXAMPLE 4.2. Define $X_{1}, X_{2}, X_{3}$ jointly distributed as follows: $\left(X_{1}, X_{2}, X_{3}\right)=$ $(1 / 2,2 / 3,1)$ with probability $1 / 3,=(1 / 2,2 / 3,0)$ w.p. $1 / 6,=(1 / 2,1 / 3,1)$ w.p. $1 / 6$, and $=(1 / 2,1 / 3,0)$ w.p. $1 / 3$. For $n>3$, let $X_{n}=X_{3}$. Then $E\left(\sup X_{n}\right)=7 / 9$, and since $X_{1}, X_{2}, \ldots$ is a martingale, $\sup \left\{E X_{t}: t \in T\right\}=E X_{1}=1 / 2$. Thus $D\left(X_{1}, X_{2}, X_{3}, \ldots\right)=5 / 18>1 / 4$.

\section{REFERENCES}

1. Y. S. Chow, H. Robbins and D. Siegmund, Great expectations: The theory of optimal stopping, Houghton Mifflin, Boston, Mass., 1971.

2. T. P. Hill and R. P. Kertz, Ratio comparisons of supremum and stop rule expectations, Z. Wahrsch. Verw. Gebiete (to appear).

3. U. Krengel and L. Sucheston, Semiamarts and finite values, Bull. Amer. Math. Soc. 83 (1977), 745-747.

4. 197-226. , On semiamarts, amarts, and processes with finite value, Adv. in Appl. Probab. 4 (1978),

School of Mathematics, Georgia Institute of Technology, Atlanta, Grorgin 30332 